\title{
Genome sequence comparison and superinfection between two related Pseudomonas aeruginosa phages, D3112 and MP22
}

\author{
Yun-Jeong Heo, ${ }^{1} \dagger$ In-Young Chung, ${ }^{1} \dagger$ Kelly B. Choi, ${ }^{1}$ Gee W. Lau ${ }^{2}$ \\ and You-Hee $\mathrm{Cho}^{1}$ \\ ${ }^{1}$ Department of Life Science, Sogang University, Seoul 121-742, Korea \\ ${ }^{2}$ Department of Pathobiology, University of Illinois at Urbana-Champaign, Urbana, IL 61802, USA
}

Correspondence

You-Hee Cho

youhee@sogang.ac.kr

Received 20 February 2007

Revised 7 May 2007

Accepted 9 May 2007

\section{INTRODUCTION}

Pseudomonas aeruginosa is an important opportunistic human pathogen that infects individuals with impaired host defences. These include patients undergoing immunosuppressive therapies and those suffering from traumatic skin damage, human immunodeficiency virus infections and cystic fibrosis (Bodey et al., 1983; Kielhofner et al., 1992; Govan \& Deretic, 1996). P. aeruginosa is a highly adaptable bacterium that forms persistent biofilms, and is resistant to most antibiotics (Costerton et al., 1999). One major concern is that cells within biofilms have a strong proclivity toward generating diverse morphotypes to enhance their ecological fitness and possibly promote strain diversity (Drenkard \& Ausubel, 2002; Boles et al., 2004). The emergence of new $P$. aeruginosa strains and the persistence of the existing antibiotic-resistant clinical isolates has led to an urgent need to explore alternative strategies for managing $P$. aeruginosa-mediated infections. Among these, bacteriophages have emerged as potentially attractive alternative anti-infection modalities (Sulakvelidze et al., 2001), as exemplified by the success of a genetically modified filamentous phage (Pf3R) in controlling experimental $P$. aeruginosa infections (Hagens et al., 2004).

Abbreviation: TFP, type four pili.

†These authors contributed equally to this work.

The GenBank/EMBL/DDBJ accession number for the complete genome sequence of MP22 is D0873690.
Many isolated $P$. aeruginosa phages are members of the viral order Caudovirales, including the families Siphoviridae (D3112 and B3), Podoviridae ( $\phi \mathrm{KMV}, \mathrm{LKA1}, \mathrm{LKD} 16$ and F116), and Myoviridae ( $\phi \mathrm{CTX}$ ). Some of these phages are useful in the genetic analyses of $P$. aeruginosa, as transposons (mini-D3112) (Darzins \& Casadaban, 1989a, b), or as integration-proficient gene delivery vectors (miniCTX) (Hoang et al., 2000). Another phage, DMS3, which is capable of generalized transduction within and between $P$. aeruginosa strains, is useful for exchange or transfer of genetic markers (Budzik et al., 2004). Moreover, the growing information on the genome sequences of these phages permits comparative genomic analyses (Braid et al., 2004; Wang et al., 2004) and comprehensive taxonomy (Rohwer \& Edwards, 2002). These new perspectives on the phylogenetic aspects of the phages may be exploited to control $P$. aeruginosa infections.

The need to further our understanding of the genetics, distribution and phylogeny of $P$. aeruginosa phages has prompted us to isolate new phages capable of infecting $P$. aeruginosa strains. Here, we report the isolation, genome sequencing and characterization of a new temperate transposable bacteriophage, MP22, from cultures of a Korean clinical $P$. aeruginosa isolate. Phage MP22 is able to form discernible plaques on $P$. aeruginosa strain PA14, a highly virulent clinical isolate that also infects hosts from multiple phylogenetic origins (Rahme et al., 2000). Phage MP22 is a double-stranded DNA bacteriophage with high similarity to phage D3112, with regard to their virion 
structures and overall genomic organization. Like D3112, MP22 requires type four pili (TFP) for infection. However, genome sequence analysis reveals that MP22 differs from D3112 in the genes encoding the phage $\mathrm{c}$ repressor and several late genes for virion morphogenesis. Expression of each $\mathrm{c}$ repressor from each phage could provide immunity to the cognate phage, but not to the non-cognate phage. Interestingly, D3112 could infect MP22 lysogens, whereas MP22 failed to infect D3112 lysogens, establishing a superinfection hierarchy between these two related phages.

\section{METHODS}

Bacterial strains, phages and culture conditions. Escherichia coli $\mathrm{DH} 5 \alpha$ and S17-1, and P. aeruginosa strains PA14, PAO1 and PAK, plus various clinical isolates (including PMM22, which carries the MP22 prophage on its genome) and their derivatives were grown at $37{ }^{\circ} \mathrm{C}$ using Luria-Bertani (LB) broth or on $2 \%$ Bacto-agar (Difco) LB or Cetrimide-agar (Fluka) plates as previously described (Heo et al., 2005). Strain PAS429, which carries the D3112cts prophage with a temperature-sensitive c repressor (cts), was kindly provided by $\mathrm{Dr}$ D.S. Guttman (Department of Botany, University of Toronto, Canada). Stationary-phase cultures were inoculated into fresh LB broth with an inoculum of $1.6 \times 10^{7}$ c.f.u. $\mathrm{ml}^{-1}$, then grown and used for experiments. Antibiotics were used at the following concentrations: for $P$. aeruginosa, rifampicin $\left(100 \mu \mathrm{g} \mathrm{ml}^{-1}\right)$, kanamycin $\left(50 \mu \mathrm{g} \mathrm{ml}^{-1}\right)$ and carbenicillin $\left(200 \mu \mathrm{g} \mathrm{ml}^{-1}\right)$; for E. coli, ampicillin $\left(50 \mu \mathrm{g} \mathrm{ml}^{-1}\right)$.

Generation of in-frame deletion mutants. All the in-frame deletion mutants in this study were created as described previously using pEX18T (Hoang et al., 1998). The designs of the oligonucleotide primers were based on sequences from $P$. aeruginosa strain PA14 (GenBank accession numbers AABQ07000000-AABQ07000005). For the pilA mutant, a $1.7 \mathrm{~kb}$ fragment encompassing the pilA gene was amplified from the PA14 chromosome. The PCR product was cloned and digested with $\mathrm{NaeI}$ followed by self-ligation to create the in-frame deletion of approximately $80 \%$ of the pilA coding region. For the flgK mutant, four oligonucleotide primers were used to amplify the $5^{\prime}$ and $3^{\prime}$ segments of the flgK gene of PA14. Approximately two-thirds of the flgK coding region was deleted by joining both amplicons using an engineered restriction site. All double-crossover deletion mutants were obtained by sucrose-resistance selection from the singlecrossover cointegrates and genotypically verified by PCR and by mutant phenotypes (e.g. loss of motility in pilA and flgK mutants), when applicable (data not shown).

Episomal gene expression. The c repressors were ectopically expressed using a multicopy plasmid, pUCP18. For the MP22 c repressor, the $1.8 \mathrm{~kb}$ left-end SalI fragment of the MP22 genome was cloned into pUCP18. For the D3112 c repressor, the $1.4 \mathrm{~kb}$ left-end fragment was amplified from strain PAS429 and cloned into pUCP18. These plasmids were introduced into PA14 or its isogenic mutants by electroporation (Choi et al., 2006).

Preparation of phage lysates. For all experiments, filtrates of phage-infected $P$. aeruginosa culture supernatant were used as phage lysates. Briefly, PAS429, PMM22 and the PA14-derived phage lysogens were grown in LB for more than $18 \mathrm{~h}$. After pelleting bacterial cells, the phage particles were precipitated from culture supernatant with $10 \%$ PEG and $1 \mathrm{M} \mathrm{NaCl}$, and dissolved in $5 \mathrm{ml}$ phage buffer [10 $\mathrm{mM} \mathrm{MgSO}_{4}, 10 \mathrm{mM}$ Tris (pH 7.6), $1 \mathrm{mM}$ EDTA], based on the method for bacteriophage $\lambda$ (Sambrook et al., 1989). Phage particles were concentrated by ultracentrifugation at $450000 \mathrm{~g}$ for $4 \mathrm{~h}$ and resuspended in phage buffer; the titre was determined prior to use.

Establishment of PA14 lysogens. Overnight, stationary-phase PA14 bacteria were used to inoculate fresh LB that contained the appropriate amount of phage lysates. After $18 \mathrm{~h}$ incubation, most bacteria were lysed and killed by the infecting phages. The survival of the remaining bacteria was due either to lysogenization of the infecting phages or to fortuitous bacterial resistance to phages. About 20 surviving colonies were plated on LB for single-colony isolation. Each purified colony was subjected to PCR using phage-specific primers. The PCR-positive colonies (six for MP22 and four for D3112) were expected to carry the prophage in their genomes and were used for further studies. PA14M and PA14D are the representative lysogens for MP22 and D3112, respectively.

Phage infection and host cell adsorption assays. Phage infection was observed by either conventional plaque assay or spotting assay (Heo et al., 2005, 2007). For plaque assay, $10 \mu \mathrm{l}$ of lysates that contained $\sim 10^{2}$ p.f.u. phages was mixed with $\sim 10^{7}$ c.f.u. of lateexponential-phase $P$. aeruginosa cells resuspended in $100 \mu$ l phage buffer. After $10 \mathrm{~min}, 3 \mathrm{ml}$ top agar was added and the mixture was plated. Plaques were visualized after $16-24 \mathrm{~h}$ of incubation at $37^{\circ} \mathrm{C}$. For spotting assay, $3 \mu \mathrm{l}$ samples of serially diluted lysates were spotted on the lawn of $P$. aeruginosa cells.

Host cell adsorption was performed as previously described (Roncero et al., 1990). Briefly, late-exponential-phase P. aeruginosa cells $\left(\sim 10^{7}\right.$ c.f.u.) were harvested by centrifugation and resuspended in phage buffer containing $\sim 10^{3}$ p.f.u. MP22. Following $30 \mathrm{~min}$ incubation at $37^{\circ} \mathrm{C}$, bacterial cells were pelleted $(13000 \mathrm{~g}$ for $10 \mathrm{~min}$ ), and the supernatants were subjected to filtration, followed by p.f.u. determination by plaque assay. The reduction in p.f.u. in the supernatants represents the amount of phage bound or inactivated by contact with the cells. The percentages of p.f.u. reduction relative to the p.f.u. reduction determined from the mixture without bacterial cells were calculated.

Transmission electron microscopy. The virion morphology of MP22 was determined by transmission electron microscopy (TEM). Formvar-coated TEM grids were subjected to hydrophilic treatment (10 min) and floated with $20 \mu \mathrm{l}$ of phage samples containing $\sim 10^{4}$ p.f.u. immediately followed by negative staining (5 s) using $20 \mu \mathrm{l} 2 \%$ uranyl acetate ( $\mathrm{pH} 4.0$ ). The grids were air-dried for $30 \mathrm{~min}$ and viewed using a transmission electron microscope (JEM 1010 EM; JEOL) at 120000-500 $000 \times$ magnification.

Extraction of phage DNA. Phage DNA was isolated according to a standard protocol (Sambrook et al., 1989). Briefly, purified phage $\left(\sim 10^{12}\right.$ p.f.u. $)$ was treated with proteinase $\mathrm{K}\left(100 \mu \mathrm{g} \mathrm{ml}^{-1}\right)$ in the presence of $0.5 \%$ SDS for $1 \mathrm{~h}$ at $65{ }^{\circ} \mathrm{C}$, extracted with phenol/ chloroform, and precipitated with ethanol. The genome size was estimated by PFGE using $1 \%$ PFGE-certified agarose in $0.5 \times$ Tris/ borate/EDTA buffer and a CHEF-DR II apparatus (Bio-Rad) as described elsewhere (Budzik et al., 2004).

Shotgun sequencing of the MP22 genome. The genome of MP22 was sequenced using an automated sequencer based on the dideoxy chain-termination method. A random genomic library was generated in the plasmid sequencing vector pUC18. In total, 361 sequencing reads from shotgun clones of about $3.0 \mathrm{~kb}$ insert size, assembled using Arachne software (http://www.broad.mit.edu/wga) and corresponding to approximately 6.41-fold coverage of the genome, were carried out. The remaining single gap, of about $1.0 \mathrm{~kb}$, was closed by direct cloning from the genomic DNA. To clearly define the end regions of MP22, we cloned and sequenced the DNA fragments from both ends of four MP22 lysogens such as PA14M. DNA fragments (300 bp) 
from both ends of the MP22 prophage were cloned into pEX18T and the resulting cointegrates of the lysogen were created via insertdirected homologous recombination. Isolated chromosomal DNA from the cointegrates was digested with $\mathrm{NruI}$ and SnaBI and then subjected to self-ligation. Subsequent sequencing of the clones that had carried over the genomic DNAs revealed the junction regions between the phage ends and the chromosome of the MP22 lysogens.

Sequence annotation and analysis. Genes that encode proteins were predicted with the GENEMARK (Isono et al., 1994), FRAME (Bibb et al., 1984), and OMIGA 2.0 (Oxford molecular) programs. The predicted translational regions have been corroborated by manual inspection of the purine rich ribosome-binding sites (Shine \& Dalgarno, 1975) and comparison with the related phage proteins. The tRNAscan-SE program was used to search for tRNA genes (Lowe \& Eddy, 1997; http://lowelab.ucsc.edu/tRNAscan-SE). The COUNTCODON program was used to determine codon usage (http://www.kazusa. or.jp/codon/countcodon.html). Slippery sequences were identified using the programmed frameshift finder program (Xu et al., 2004; http://chainmail.bio.pitt.edu/ junxu/webshift.html). BLAST programs such as BLASTN, BLASTX and BLASTP (http://www.ncbi.nlm.nih.gov/ blast), were used for similarity searches. Multiple sequence alignments were generated with CLUSTAL W (Thompson et al., 1994; http:// www.ebi.ac.uk/clustalw). Protein motifs were scanned at the PROSITE server (http://www.expasy.org/prosite), and DNA motifs were examined with OMIGA software. Genome alignment was performed using the MAUVE program at its default setting (Darling et al., 2004; http:// gel.ahabs.wisc.edu/mauve). Dot-plot comparison and other general sequence manipulations, including primer design and restriction-site search, were performed using the OMIGA program.

PCR sequencing of the D3112 regions. To amplify the D3112 ORF17 and the junction regions between ORF46 and ORF47, appropriate oligonucleotide primer pairs were designed based on the annotation information for the D3112 genome (Wang et al., 2004). Each $0.5 \mathrm{~kb}$ DNA fragment was amplified and directly sequenced using the same primers.

\section{RESULTS AND DISCUSSION}

\section{Identification and isolation of phage MP22}

During the course of our studies on pyocin profiling, we discovered some phage-derived killing activities from culture supernatants of our pyocin-producing $P$. aeruginosa strains (Heo et al., 2005). Apart from three wellcharacterized strains, PA14, PAO1 and PAK, an additional $28 P$. aeruginosa clinical isolates (designated PMM1 to PMM28) were analysed for phage-derived killing activities. Culture supernatants derived from PMM15 and PMM22 were found to kill PA14, with the killing by the PMM22 supernatant being more apparent (data not shown). Increasingly diluted culture supernatant of PMM22 showed decreasing numbers of discrete plaques on PA14, suggesting that the killing activity is associated with phages, since non-replicative killing activities such as pyocins show a diffuse thinning of cell growth, resulting in less marked plaques (Daw \& Falkiner, 1996). This phage was purified from a single plaque and designated MP22. Purified and amplified MP22 was shown to form plaques on PA14 cells (see below).

\section{Morphology of phage MP22: a $\lambda$-like phage}

We determined the morphology of MP22 phage particles by transmission electron microscopy. MP22 has a flexible tail $\sim 150 \mathrm{~nm}$ in length and an icosahedral head $\sim 50 \mathrm{~nm}$ in diameter (Fig. 1). This morphology is similar to the virion structure of the $\lambda$-like phages of the Siphoviridae family, which have characteristic isometric heads and long, flexible, non-contractile tails.

\section{Receptor of phage MP22: type IV pili are required for host cell adsorption}

A number of surface receptors on $P$. aeruginosa have been implicated as bacteriophage receptors. In particular, the type IV pili (TFP) have been shown to function as receptors for various $P$. aeruginosa phages, including filamentous phage Pf (Bradley, 1973b), single-stranded RNA phage PP7 (Bradley, 1967), Bradley B-type phage PO4 (Bradley, 1973a), and temperate transducing phages such as F116 (Byrne \& Kropinski, 2005), DMS3 (Budzik et al., 2004), B3 (Roncero et al., 1990) and D3112 (Wang et al., 2004). To determine whether TFP serve as receptors for MP22, we created a deletion mutant of the TFP gene, pilA. An unrelated deletion in $f l g K$, which encodes a component of the flagellum, another important extracellular appendage of $P$. aeruginosa, was included as a negative control. As shown in Fig. 2, whereas MP22 could form discrete plaques on wild-type PA14 and flgK bacteria, no such plaques were formed on pilA bacteria. Furthermore, the ability of MP22 to adsorb to the pilA bacteria was drastically reduced: the percentages of phages bound to the wild-type PA14 and flgK bacteria were $73.9 \%$ and $74.2 \%$, respectively in our experimental conditions, whereas the percentage of phages bound to the pilA mutant was $12.2 \%$ (data not shown). This low percentage of viral adsorption to pilA mutant cells may be due to non-specific interaction between MP22 phages and pilA cells or to the presence of a secondary receptor other than TFP. Because MP22 was unable to form plaques on pilA mutant cells under any conditions observed (data not shown), it is concluded that $P$. aeruginosa TFP are the primary receptors for phage MP22 entry into PA14 cells.
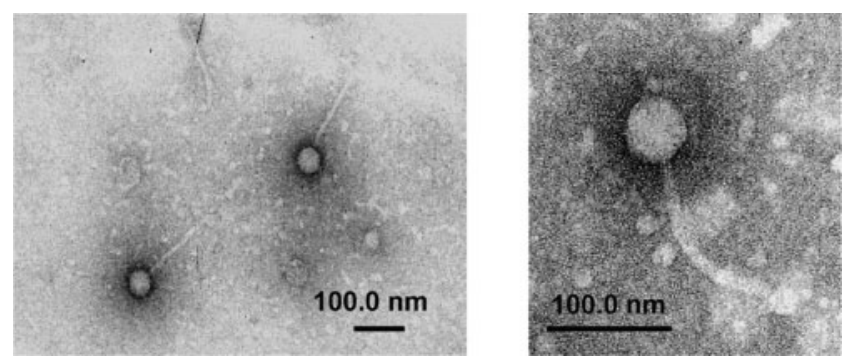

Fig. 1. Transmission electron micrographs of phage MP22 negatively stained with uranyl acetate, revealing its virion structure and flexible tail. 


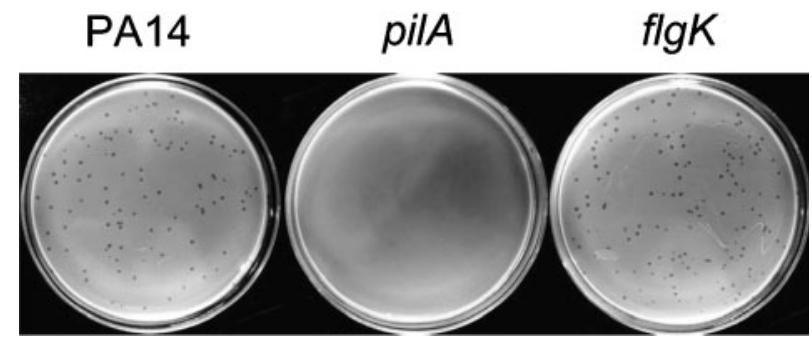

Fig. 2. Type IV pilus of $P$. aeruginosa is the receptor for phage MP22. Plaque formation. Approximately $10^{2}$ p.f.u. of phage MP22 was mixed with $\sim 10^{7}$ c.f.u. of $P$. aeruginosa wild-type (PA14) or its isogenic mutants ( $p i l A$ or $f / g K)$. Plaques were visualized after 16-24 h of incubation at $37^{\circ} \mathrm{C}$.

\section{Genome of phage MP22: a double-stranded linear DNA of 36409 bp}

To investigate the nature of the MP22 genome, it was isolated from purified phage particles. The isolated phage nucleic acid was insensitive to RNase A and S1 nuclease, but susceptible to restriction by common DNA restriction enzymes such as AluI and HaeIII (data not shown), indicating that MP22 is a double-stranded DNA phage. PFGE analysis estimated the MP22 genome size to be 36$40 \mathrm{~kb}$ (data not shown). We determined the complete genomic sequence of MP22 by a shotgun sequencing approach. A total of 361 DNA sequencing reads were achieved, providing, on average, 6.41 -fold coverage of the genome. The linear genome is composed of $36409 \mathrm{bp}$ (GenBank accession no. DQ873690), which is in agreement with the PFGE estimate. The mol\% G+C content and codon usage analyses revealed no significant difference between MP22 and $P$. aeruginosa PAO1, implying that MP22 is a generic $P$. aeruginosa phage (Table 1 and data not shown). Unlike several members of the Siphoviridae, which possess tRNA genes that can increase the overall translation rate by transcribing tRNA that recognizes rare codons (Kropinski, 2000), no tRNA species was found on the MP22 genome, as determined by tRNAscan analysis (Lowe \& Eddy, 1997).

\section{Characterization of the MP22 genome ends}

Sequencing of the leftmost and the rightmost ends of MP22 revealed heterogeneous $P$. aeruginosa sequences (data not shown). This suggests that MP22 is capable of integrating into different locations on the $P$. aeruginosa genome as in other P. aeruginosa phages such as D3112 and B3 (Braid et al., 2004; Wang et al., 2004). Therefore, instead of trimming the heterogeneous sequences, we directly cloned both left- and right-end regions from the genome of one established MP22 lysogen (PA14M). PA14M prophage was found to have integrated into the intergenic region between lpxO1 (PA4512) and piuB (PA4513) (data not shown). This direct cloning and sequencing has enabled us to define both junction sequences between the PA14 and MP22 genomes, and thus determine the left and right ends of the MP22 prophage, which are $5^{\prime}$-TGT- $3^{\prime}$. Because sequence variations as well as imprecise excision could occur during prophage excision (Darzins \& Casadaban, 1989b; Wang et al., 2004), our approach may be a valid method to determine the functional MP22 genome, based on the observation that a functional phage lysate could be obtained from the established lysogen PA14M (see Fig. 7).

\section{Genome organization of MP22}

DNA sequence comparison using BLASTN analysis revealed that the genome of MP22 is highly similar to that of bacteriophage $\mathrm{D} 3112$, with conserved genomic and synteny characteristics (Fig. 3). Based on similarity to D3112 and other related phages (Braid et al., 2004; Wang et al., 2004; Marrs \& Howe, 1990), the genome of MP22 can be divided into three functional regions: early, middle and late regions (Figs 3 and 4). The early or left-end region is responsible for genome integration, modulation of phage gene expression and modulation of host response. The small middle region is responsible for controlling the transcription of the late genes. The late or right-end region is responsible for virion morphogenesis. Lysis proteins exist in the late regions of many Siphoviridae family phages, but we could not identify the lysis proteins of MP22 or D3112, based on homology searches. Lysis cassettes in bacteriophages of Gram-negative bacteria consist of a holin, an antiholin, an endolysin and Rz/Rzl proteins, but they display little sequence homology to other proteins of the same functional classes (Wang et al., 2000). Identification of the lysis cassettes of MP22 requires further investigation based on domain-level identification (Summer et al., 2004) as well as experimental verification.

Putative ORFs within the MP22 genome were identified using the GENEMARK (Isono et al., 1994), FRAME (Bibb et al., 1984) and OMIGA programs. The predicted protein sequences were used for BLASTP analysis to identify ORFs displaying significant homology with other bacteriophage or bacterial proteins. The nucleotide sequence of each MP22 ORF was also subjected to BLASTX analysis to screen for homologues within the MP22 genome. Sequences upstream (within 4-14 bases upstream of potential start codons) and in the early portion of each predicted ORF were examined manually for the presence of a conserved purine-rich ribosome-binding site (AGGAGG) (Ma et al., 2002). The sequence was also examined for the close proximity of stop codons at the end of one gene and start codons immediately downstream from the stop codons or upstream of another gene; this positioning is frequently observed in prokaryotic operons (Ma et al., 2002). When multiple possible ORFs were present, the most likely coding regions were chosen based on codon usage and high mol\% G $+\mathrm{C}$ content of the third codon position, as aided by the FRAME program. We also looked for the programmed frameshifts that are widespread in many bacteriophages 
Table 1. Proposed coding regions of phage MP22

\begin{tabular}{|c|c|c|c|c|c|}
\hline Gene $^{*}$ & Range $(b p) \dagger$ & $\begin{array}{l}\text { Size } \\
(\mathbf{a a})\end{array}$ & $\begin{array}{c}\operatorname{mol} \% \\
G+C \ddagger\end{array}$ & Highest similarity gene or putative function & $E$ value $\S$ \\
\hline ORF1 & $170-799$ & 236 & $\mathbf{5 7 . 2}$ & D3112p01; phage repressor, c & $5 \times 10^{-61}$ \\
\hline ORF3 & $1423-1857$ & 144 & 65.7 & D3112p03; hypothetical protein & $1 \times 10^{-65}$ \\
\hline ORF5 & $1857-2066$ & 69 & 62.8 & D3112p05; hypothetical protein & $2 \times 10^{-08}$ \\
\hline ORF6 & $2059-4131$ & 690 & 67.2 & D3112p06; DNA transposition protein & 0 \\
\hline ORF8 & $4895-5542$ & 215 & 64.3 & D3112p08; hypothetical protein & $9 \times 10^{-97}$ \\
\hline ORF10 & $5527-5643$ & 38 & 66.7 & D3112p10; hypothetical protein & 5.1 \\
\hline ORF11 & $5643-6056$ & 137 & 67.4 & D3112p11; hypothetical protein & $3 \times 10^{-60}$ \\
\hline ORF12 & $6046-6330$ & 94 & 67.0 & D3112p12; hypothetical protein & $2 \times 10^{-41}$ \\
\hline ORF13 & $6347-7243$ & 298 & 62.6 & D3112p13; host nuclease inhibitor protein, Gam; & $3 \times 10^{-90}$ \\
\hline ORF14 & $7245-7553$ & 102 & 62.1 & D3112p14; hypothetical protein & $4 \times 10^{-40}$ \\
\hline ORF19 & $9074-9523$ & 149 & 66.0 & D3112p19; putative integral membrane protein & $2 \times 10^{-64}$ \\
\hline ORF19a\| & $9627-9974$ & 115 & 66.4 & None & \\
\hline ORF20 & $9919-10389$ & 156 & 65.8 & D3112p20; hypothetical protein & $2 \times 10^{-63}$ \\
\hline ORF21 & $10389-10805$ & 138 & 65.5 & D3112p21; putative structural protein & $2 \times 10^{-60}$ \\
\hline ORF21a & $10805-11047$ & 80 & 63.8 & None & \\
\hline ORF22 & $11044-11445$ & 133 & 68.4 & D3112p22; hypothetical protein & $6 \times 10^{-40}$ \\
\hline ORF23 & $11556-11744$ & 62 & 65.6 & D3112p23; hypothetical protein & $8 \times 10^{-17}$ \\
\hline ORF24 & $11753-12253$ & 166 & 66.7 & D3112p24; hypothetical protein & $8 \times 10^{-69}$ \\
\hline ORF269 & $12250-14025$ & 591 & 61.6 & Putative phage protein [Burkholderia vietnamiensis] & 0 \\
\hline ORF27 & $14035-15615$ & 526 & 65.9 & D3112p27; portal protein & 0 \\
\hline ORF28 & $15615-16901$ & 428 & 66.9 & D3112p28; head assembly cofactor & 0 \\
\hline ORF37 & $21174-21341$ & 55 & 64.2 & D3112p37; hypothetical protein & 0.007 \\
\hline ORF38 & $21338-21754$ & 138 & 61.1 & D3112p38; hypothetical protein & $7 \times 10^{-47}$ \\
\hline ORF39 & $21751-22224$ & 157 & 64.3 & D3112p39; hypothetical protein & $2 \times 10^{-57}$ \\
\hline ORF40 & $22212-22397$ & 61 & 57.9 & $\mathrm{D} 3112 \mathrm{p} 40$; hypothetical protein & $4 \times 10^{-09}$ \\
\hline ORF41 & $22423-23196$ & 257 & 62.3 & D3112p41; hypothetical protein & $7 \times 10^{-63}$ \\
\hline ORF42 & $23200-23697$ & 165 & 65.5 & D3112p42; tail assembly chaperone, gpG & $2 \times 10^{-22}$ \\
\hline ORF43\# & $23200-23822$ & 207 & 64.7 & D3112p43; tail assembly chaperone, gpGT & 0.58 \\
\hline ORF45e & $23824-27522$ & 1232 & 65.9 & B3ORF49; tail length tape measure protein, gpH [phage B3] & $3 \times 10^{-177}$ \\
\hline ORF46es & $27530-28486$ & 318 & 64.5 & B3ORF50; hypothetical protein [phage B3] & $1 \times 10^{-172}$ \\
\hline ORF48 & $28488-29411$ & 307 & 62.2 & B3ORF51; hypothetical protein [phage B3] & $3 \times 10^{-156}$ \\
\hline ORF49 & $29411-31114$ & 567 & 66.1 & D3112p49; hypothetical protein & 0 \\
\hline ORF50 & $31149-31925$ & 258 & 64.3 & D3112p50; hypothetical protein & $2 \times 10^{-138}$ \\
\hline ORF51 & $31934-32173$ & 79 & 59.1 & D3112p51; hypothetical protein & $5 \times 10^{-26}$ \\
\hline ORF52 & $32170-32388$ & 72 & 65.3 & D3112p52; hypothetical protein & $8 \times 10^{-29}$ \\
\hline ORF53 & $32378-34585$ & 735 & 65.4 & D3112p53; hypothetical protein & 0 \\
\hline ORF54 & $34582-35733$ & 383 & 62.0 & D3112p54; hypothetical protein & 0 \\
\hline ORF55 & $35730-36020$ & 96 & 66.3 & D3112p55; hypothetical protein & $6 \times 10^{-36}$ \\
\hline
\end{tabular}

${ }^{\star}$ Gene numbers for MP22 correspond to the gene numbers of D3112 (GenBank accession number AY304005). For genes that have not been identified on the D3112 genome, suffixes ( $\mathrm{a}$ and b) are used. Suffix e (extended) is used for genes that span two D3112 genes. Genes in the dissimilarity regions (except for region 3 ) are indicated in bold. 
Table 1. cont.

$\dagger$ Range from the first nucleotide of the initiation codon to the third nucleotide of the stop codon. Only one gene (ORF1) has the leftward orientation on the genome sequence.

$\ddagger$ Significant deviations $(P<0.01 ;$ t-test $)$ from the average $(64.0 \%)$ are shown in bold.

§BLASTP values were generated at the URL http://www.ncbi.nlm.nih.gov/blast using composition-based statistics.

IIORF19a was also identified in phage D3112 with 92.2\% identity; it was not identified in the previous report (Wang et al., 2004).

SGenes whose homologues in phage D3112 are less similar than the following ORFs: putative protein from Burkholderia vietnamiensis G4 (ZP_00423247); conserved hypothetical protein from Neisseria meningitidis Z2491 (CAB85070); ORF49, ORF50 and ORF51 from bacteriophage B3 (GenBank accession no. NC_006548).

\#ORF43 contains the overlapping slippery sequence (TTTTTTC at 23 556-23 562) within ORF42 (gpG) that could shift - 1 frame (Xu et al., 2004) and display similarity also to D3112p42 (gpG) at BLASTP value of $2 \times 10^{-2}$.

(Xu et al., 2004). In phage $\lambda$, the two tail genes ( $G$ and $T$ ) that precede the tail length tape measure gene $(\mathrm{H})$ are translated as a pair of overlapping proteins called gpG and gpGT, where gpGT is produced by a programmed translational frameshift (Levin et al., 1993). It has been found that this arrangement of the three genes is widely conserved among long-tailed phages, even in the absence of any detectable sequence similarity (Xu et al., 2004). The frameshifts are usually of the -1 type and occur at a characteristic 'slippery sequence'. By using the programmed frameshift finder program (Xu et al., 2004), we identified the overlapping slippery sequence (TTTTTTC at 23 556-23 562) within ORF42 (gpG) that could shift -1 frame, which might result in ORF43 (gpGT). The same slippery sequence was also identified within the D3112 ORF42 (TTTTTTC at 25008-25 014) (data not shown).

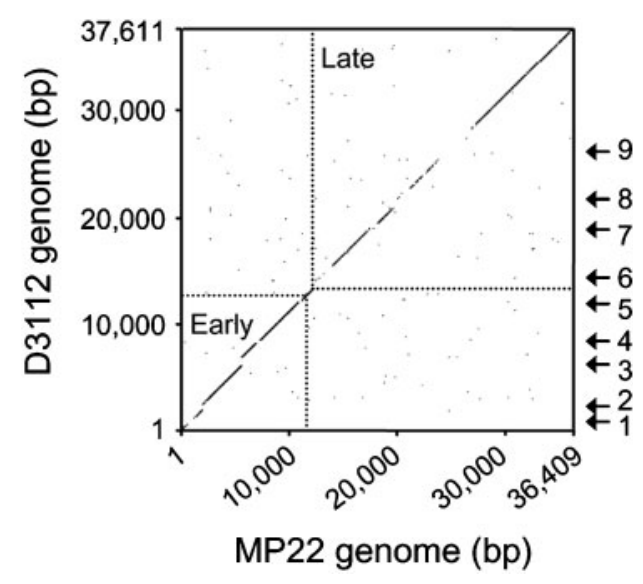

Fig. 3. Dot-plot comparison of MP22 and D3112 genome sequences. The dot-plot was generated using the OMIGA 2.0 program (Oxford Molecular) with window size 30 and hash value 6 . Dots are generated with a minimum percentage score of 65 . Dotted lines denote the approximate boundaries of the early and late regions of both phages. The nine lesions (indicated by arrows) in the diagonal line represent regions of genomic dissimilarity between MP22 (GenBank accession no. DQ873690) and D3112 (GenBank accession no. AY394005).
The above analyses enabled us to identify a total of 51 potential ORFs in the genome of MP22 (Table 1). Four potential translational initiation codons were identified: AUG (46 ORFs), GUG (ORF38, 39, and 50), CUG (c repressor) and UUG (ORF12). Because ORF12 encodes a relatively short ORF (94 aa), with a rare initiation codon (UUG), it is also likely that this gene is a non-functional gene remnant which has been generated during genome evolution. Whether ORF10 encodes a protein product or not remains to be experimentally elucidated.

\section{Genome and coding region comparisons between phages MP22 and D3112}

The BLASTP search results showed that a majority of the MP22 genes (48 out of 51), with the exception of ORF21a, $29 \mathrm{a}$ and $29 \mathrm{~b}$, are similar to genes in D3112. The ORFs of MP22 are named ORFs 1-55, with the number chosen to correspond to the orthologous ORF of phage D3112. The identity of the highest scoring homologues and their putative functions are summarized in Table 1.

Based on the direct nucleotide sequence comparison between MP22 and D3112, major 'relative' differences were identified in nine separate regions, as illustrated in the genomic dot-plot and the comparative synteny maps (Figs 3 and 4). Regions 1, 2, 4, 6, 8, and 9 exhibit dissimilarity between ORFs present in both phages. Regions 2, 3, 6, 7, 8 and 9 also contain D3112-unique ORFs (blue ORFs in Fig. 4: ORF4, 9, 25, 30, 31, 34 and 44) which are missing in MP22. Regions 5 and 7 contain the MP22-specific ORFs (red ORFs in Fig. 4: ORF21a, 29a and 29b). These three MP22-specific ORFs are very small in size and display no significant sequence homology to known proteins in the database (Table 1).

Dissimilarity regions 2, 6, 8 and 9 contain multiple sequence variations, including strain-specific ORFs. This could be caused by gene gain or loss that affects the nearby ORFs in those dissimilarity regions, or the en bloc gene transfer of two or more functionally associated ORFs. As a whole, the genome and coding region comparison shows the overall synteny between these phages. Nonetheless, some profound dissimilarities are noticeable in the late region. These divergences in the late genes may have caused 


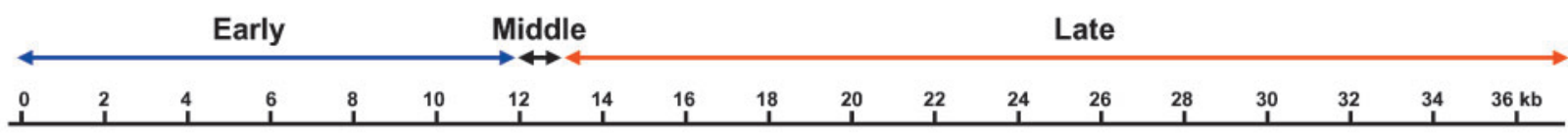

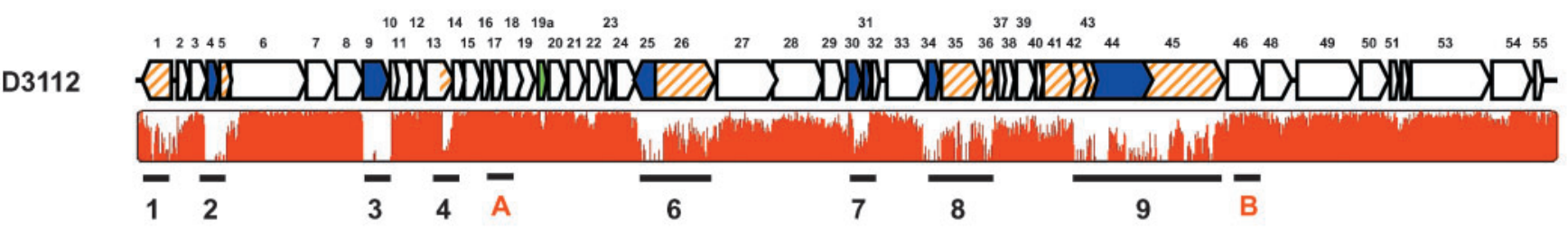

MP22

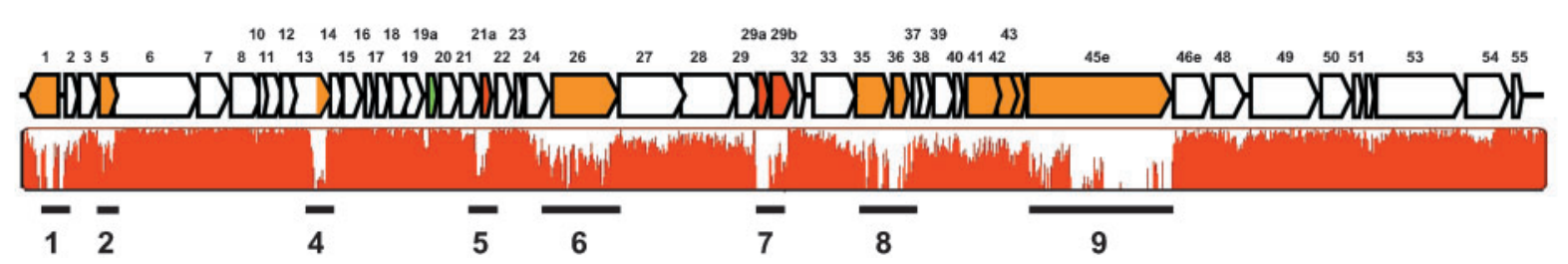

Fig. 4. Comparative genome maps with genome alignment results. The genomes and putative coding regions of D3112 and MP22 are drawn in parallel approximately to scale. The 55 ORFs from D3112 and the 51 ORFs from MP22 are shown as boxes and gene numbers. The relative locations of orthologous genes, shown by open boxes, reveal a high degree of synteny (see Table 1 for details). The approximate boundaries of the early, middle and late regions are indicated by lines with double arrowheads. The genome alignment result generated by the MAUVE program at its default settings is indicated below each genome map, showing the degree of nucleotide similarity between D3112 and MP22 (Darling et al., 2004). The ORF colours are as follows: white, present in both phages; blue, present in D3112 only; red, present in MP22 only; orange in MP22 and hatched orange in D3112 represent relatively low similarity between the genes; green, newly identified gene (ORF19a; see Table 1 for details). Regions 1-9 represent the genomic dissimilarity regions designated in Fig. 3. Regions A (ORF17) and B (MP22 ORF46e) contain the update for the D3112 genome sequence (see Fig. 5 for details). All the genes are transcribed rightward, except for both c repressors and D3112 ORF25.

subtle differences in virion structure between MP22 and D3112, and possibly contribute to their distinct host recognition and host range.

\section{Dissimilar ORFs between phages MP22 and D3112}

We have calculated the percentage identities and similarities between the ORFs of synteny, based on protein sequence homologies. Proteins in dissimilarity regions 3, 5 and 7 do not show significant dissimilarity to the corresponding D3112 proteins (more than $85 \%$ identity and $90 \%$ similarity; data not shown), indicating functional conservation during the genetic changes in these dissimilarity regions. In contrast, proteins in dissimilarity regions $1,2,6,8$ and 9 display relatively lower similarity to the corresponding proteins (less than $60 \%$ identity and $80 \%$ similarity). The most dissimilar ORFs in these dissimilarity regions are c repressor (ORF1), ORF5, 26, 35 and 45e. ORF13s in dissimilarity region 4 display $73.7 \%$ identity and $86.5 \%$ similarity (data not shown). Interestingly, the first 176 amino acids in the $\mathrm{N}$-terminus of both ORF13s are almost identical $(173 / 176=98.3 \%$ identity; data not shown) to each other, and to Gam proteins from $\mathrm{Mu}$ or related phages, which are usually 176 aa long. In contrast, the ORF13s from MP22 and D3112 are 298 and 266 aa, respectively. The C-terminal region of MP22 ORF13 displays no significant homology to proteins in the current database. However, the C-terminal region of D3112 ORF13 exhibits homology $(69.6 \%$ similarity with BLASTP E value of $5 \times 10^{-8}$ ) to a small (93 aa) hypothetical protein of a pathogenic E. coli (ECs4949 of NP_312976), the significance of which remains to be determined. It is possible that both Gam proteins of phages D3112 and MP22 have a modular structure with an additional function at their Cterminal domain.

Our analysis indicates that ORF45e of MP22 is an extended version of D3112 ORF45. The sequence similarity between MP22 ORF45e (1232 aa) and D3112 ORF45 (720 aa) is located at the $\mathrm{C}$-terminus, whereas the $\mathrm{N}$-terminus of ORF45e is associated with COG5281, a phage-related minor tail protein. We noticed that D3112 ORF44 has been annotated as the tail tape measure protein $(\mathrm{gpH})$ of D3112, which is also associated with COG5281 (data not shown). A comparison of the tails of D3112 and MP22 showed that they appear to be of similar length, so they should have tail tape measure protein genes of roughly similar size. Furthermore, gpH is usually the longest ORF of a typical Siphoviridae phage. Based on these considerations, it is possible that the D3112 tail tape measure protein was split 
into two parts, i.e. 514 aa ORF44 and 720 aa ORF45, perhaps due to a sequencing error. A single $\mathrm{D} 3112 \mathrm{gpH}$ of about 1230 aa is likely to be encoded by what has been annotated as those two genes.

ORF46e (318 aa) is most homologous to ORF50 (318 aa) of phage $\mathrm{B} 3$ (Table 1). Interestingly, the $\mathrm{N}$-terminus of MP22 ORF46e is homologous to D3112 ORF46 (183 aa) whereas the C-terminus of MP22 ORF46e is homologous to D3112 ORF47 (146 aa). A careful comparison of DNA sequences at the junction between D3112 ORF46 and ORF47 with the corresponding region within ORF46e of MP22 revealed a nucleotide (A) addition in the D3112 sequence, in addition to the putative polymorphic variations (Fig. 5a). Suspecting that this (A) insertion in D3112 was a sequencing error that split the original ORF into ORF46 and 47, we determined the nucleotide sequence of the corresponding region directly amplified from strain PAS429; the sequence clearly verified the sequencing error in the previous study (Wang et al., 2004). The corrected sequence of ORF46 for D3112 is shown in Fig. 5(b).

Another misannotation, in D3112 ORF17, has been found and corrected. The published version of D3112 ORF17 is 60 aa with a weak ribosome-binding signature (Wang et al., 2004). In contrast, ORF17 of MP22 is 98 aa with a conserved ribosome-binding signature (Fig. 5c). The discrepancy appears to be caused by different annotation of the initiation codon between ORF17 of D3112 and MP22 (Fig. 5c). We suspected that a sequencing error had occurred and thus resequenced the PCR-amplified ORF17 from D3112. As shown in Fig. 5(c), the amino acid sequence of the corrected D3112 ORF17 is $100 \%$ identical to that of the MP22 ORF17, with a conserved ribosomebinding sequence (GAGCAGC- $\mathrm{N}_{5}-\mathrm{ATG}$ ). The ORF17s only differ at four nucleotides along the entire coding sequences and these are caused by conservative substitutions, resulting in no amino acid changes (Fig. $5 \mathrm{c}$ ). These analyses reveal that comparative annotations of related genome sequences based on codon usage, ribosome binding sequences, and third codon mol\% G $+\mathrm{C}$ content enabled us to find the sporadic sequence errors arising in large-scale genomic sequencing projects.

The least similar conserved protein, ORF35 (304 aa), encodes a capsid protein for head morphogenesis (COG4397). ORF35 is similar to the major head subunit in phage Mu. However, the closest homologue of ORF35 in the current database is not the D3112 ORF35, but a hypothetical protein (NMA1847) of Neisseria meningitidis Z2491 (Table 1), with a BLASTP $E$ value of $3 \times 10^{-108}$. The ORF35 of MP22 has lower BLASTP $E$ value $\left(4 \times 10^{-29}\right)$ towards the ORF35 of D3112. Careful analysis at the nucleotide as well as the amino acid sequence level led us to conclude that the virion-morphogenesis proteins of MP22 are more diverged from D3112 than are other regions. This particularly high dissimilarity in virion capsid proteins implies that the evolutionary divergence may be due to easier horizontal gene transfer and/or a faster mutation rate (a)

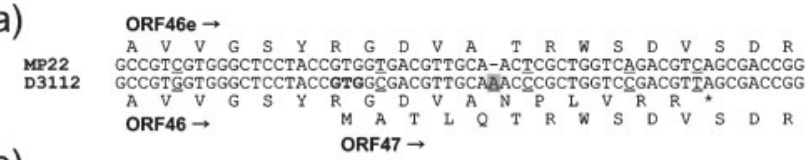

(b)

D3112 GCCGTGGTGGGCTCCTACCGTGGCGACGTTGCA-ACCCGCTGGTCCGACGTTAGCGACCGG ORF46 $\rightarrow$

(c)

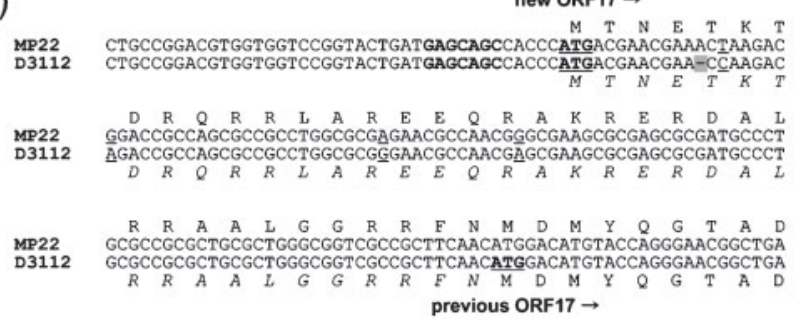

Fig. 5. Nucleotide sequence comparison of ORF46e and ORF17 between the MP22 and D3112 genomes. (a) Nucleotide sequences at residues 28028-28087 of the ORF46e region of the MP22 genome (60 nt) and 29338-29398 of the D3112 genome $(61 \mathrm{nt})$ and their translated amino acid sequences are shown (see region $B$ in Fig. 4). A partial amino acid sequence of each ORF (ORF46e, ORF46 and ORF47) is indicated with the initiation codon (GTG; bold) of the D3112 ORF47. Single nucleotide differences between the MP22 and D3112 sequences are underlined. The shaded $A$ residue indicates a sequencing error in the D3112 genome sequence (see Results for details). (b) The corrected nucleotide sequences at residues 29338-29 398 of the original version of the D3112 genome sequence. The AAA sequence was verified to be $A A$, which results in the same amino acid sequences for ORF46e and ORF46 of D3112. Underlining indicates nucleotides different from those in MP22. (c) Nucleotide sequences in the ORF1 7 coding region at residues 8347-8526 of the MP22 genome (180 nt) and 9471-9649 of the D3112 genome (179 nt) are shown, along with the translated amino acid sequences for the $\mathrm{N}$-terminal region (see region $\mathrm{A}$ in Fig. 4) and the ATG initiation codons (bold and underlined). Single-nucleotide differences between the MP22 and D3112 sequences are underlined. The shaded nucleotide missing from D3112 indicates the sequencing error. The new initiation codon (ATG; bold and underlined) and the additional amino acids (italic) of the D3112 ORF17 are indicated (new ORF17), with the potential ribosomebinding sequences (GAGCAGC) shown in bold.

compared to the rest of the genes within the phage genomes. This may ultimately lead to functional diversification of these phages in host recognition and survival in the natural environment.

Besides the late region, the dissimilarity in ORF1 between phages MP22 and D3112 is noteworthy. The ORF1 of both phages encodes the $\mathrm{c}$ repressor, which leads to repression of all transcription units (Krylov et al., 1985). As shown in Fig. 6, one of the striking dissimilarities between the two c repressors lies within the second helix (corresponding to $\alpha 3$ in $\lambda \mathrm{cI}$ repressor) of the proposed helix-turn-helix DNA 
binding motif (QG vs ET). These residues are purported to be involved in direct contact with the base pairs and hence to be important in sequence-specific recognition of the phage operator, as for the $\lambda \mathrm{cI}$ repressor (Pabo \& Sauer, 1992). The dissimilarity in the $c$ repressors and its implication in cross-immunity are further discussed below.

\section{The $c$ repressor divergence is associated with the absence of cross-immunity}

Deletion of D3112 ORF1, which encodes the c repressor, derepresses transcription of all the phage genes (Salmon et al., 2000). We have shown that the important amino acid residues within the DNA-binding domain of MP22 and D3112 c repressors differ markedly (Fig. 6). Therefore, we hypothesized that $P$. aeruginosa cells could be protected from infection by MP22 or D3112 phages by the introduction of the phages' own cognate $c$ repressor, but not by $\mathrm{c}$ repressor from the other phage. Fig. 7(a) shows that PA14 cells harbouring the multicopy plasmid-derived c repressor from MP22 ( $\left.\mathrm{c}_{\mathrm{MP} 22}\right)$ could resist infection by MP22 lysates either from the original strain (PMM22) or from the lysogenized PA14 strain (PA14M), but were susceptible to infection and lysis by D3112 lysates (PA14D and PAS429) (Fig. 7a, middle strip). Likewise, PA14 cells containing the $\mathrm{c}$ repressor from $\mathrm{D} 3112\left(\mathrm{c}_{\mathrm{D} 3112}\right)$ were immune to D3112 infection but susceptible to MP22 infection (Fig. 7a, lower strip). The pUCP18 control plasmid did not affect phage-susceptibility (data not shown). Phage adsorption assay revealed that the phages were able to adsorb to PA14 cells expressing either c repressor gene (data not shown). These results suggest that the immunity to infection by cognate phages was due to transcription repression by $\mathrm{c}$ repressors already present in the host cells. The absence of the cross-immunity indicates that the $\mathrm{c}$ repressors within the host cells are unable to confer resistance to non-cognate phages. This result suggests that, despite high similarity in their genomic and morphological characteristics, MP22 and D3112 are in different immunity groups as a result of the dissimilarity between their $\mathrm{c}$ repressors.

\section{Superinfection hierarchy between D3112 and MP22}

During the superinfection experiment, we observed that the PA14M cells (lysogenized with MP22 phage) allowed superinfection by D3112, as did PA14 expressing the $\mathrm{c}$ repressor from MP22 ( $\left.\mathrm{c}_{\mathrm{MP} 22}\right)$ (Fig. 7a). Intriguingly, PA14D cells, which are D3112 lysogens, did not allow growth of MP22 (Fig. 7b). It is likely that gene(s) unique to D3112 may act alone or synergistically with c repressor to confer superinfection hierarchy (D3112>MP22) and protect host cells harbouring D3112 (PAS429 and PA14D) against superinfection by MP22. Alternatively, the gene(s) may affect the properties of the TFP receptor and/or cell surface of the D3112 lysogens in a way that hinders the adsorption by superinfecting phages. The

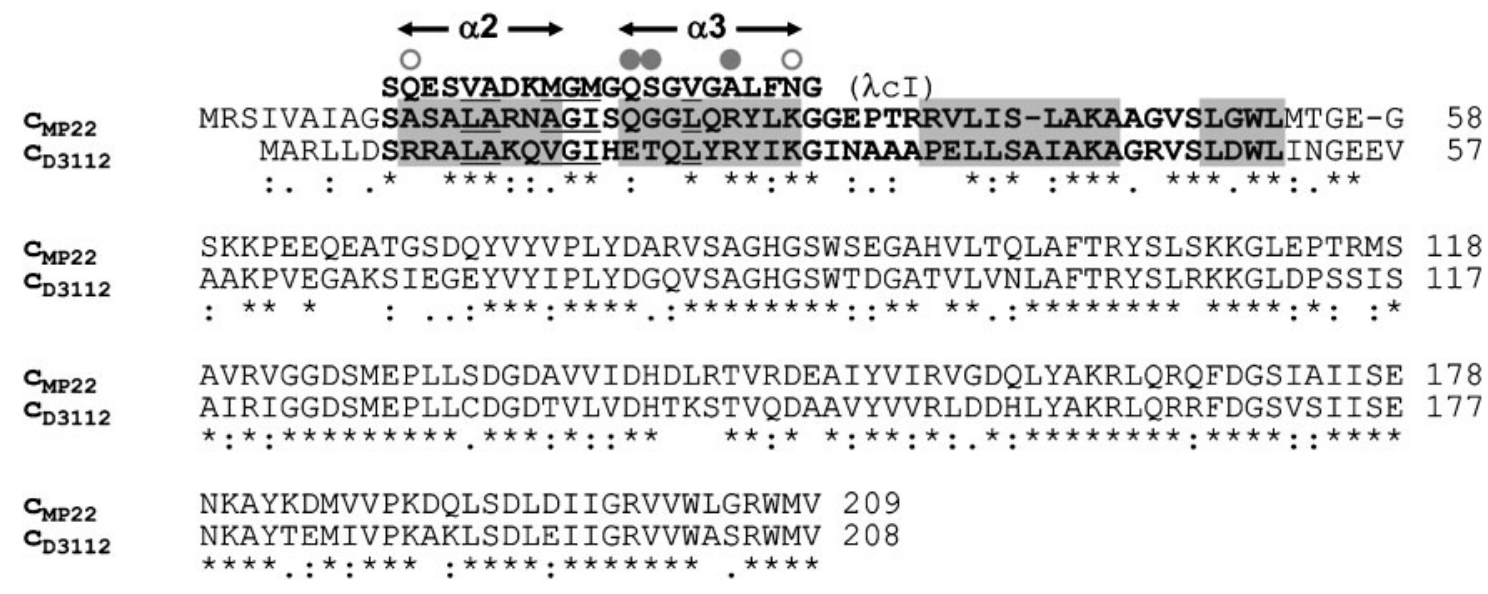

Fig. 6. The DNA-binding helix-turn-helix motif of MP22 c repressor is different from that of D3112. Alignment of both $\mathrm{c}$ repressor proteins ( $\mathrm{C}_{\mathrm{MP} 22}$ and $\mathrm{C}_{\mathrm{D} 3112}$ ) is shown with the initiation codon of the D3112 c repressor, according to previous research by Salmon et al., (2000). The sequence alignment of full-length protein sequences was generated with CLUSTAL W. Conservation of identical residues is designated by asterisks. Colons and dots indicate the conservation of similar residues based on the Gonnet PAM250 matrix similarity scores: colons, strong similarity; dots, weak similarity (Thompson et al., 1994). Four putative $\alpha$-helices are shaded, which are included in the phage repressor motif (PS50943) in the PROSITE database. The putative DNA binding helix-turn-helix motif is represented with the corresponding motif of the $\lambda$ cl repressor above ( $\alpha 2$ and $\alpha 3$ helices) (Pabo \& Sauer, 1992). Conserved features are marked: small hydrophobic residues are underlined, residues interacting with the DNA phosphate backbone are indicated by open circles, and residues in the $\alpha 3$ helix required for sequence-specific DNA binding are indicated by filled circles. 


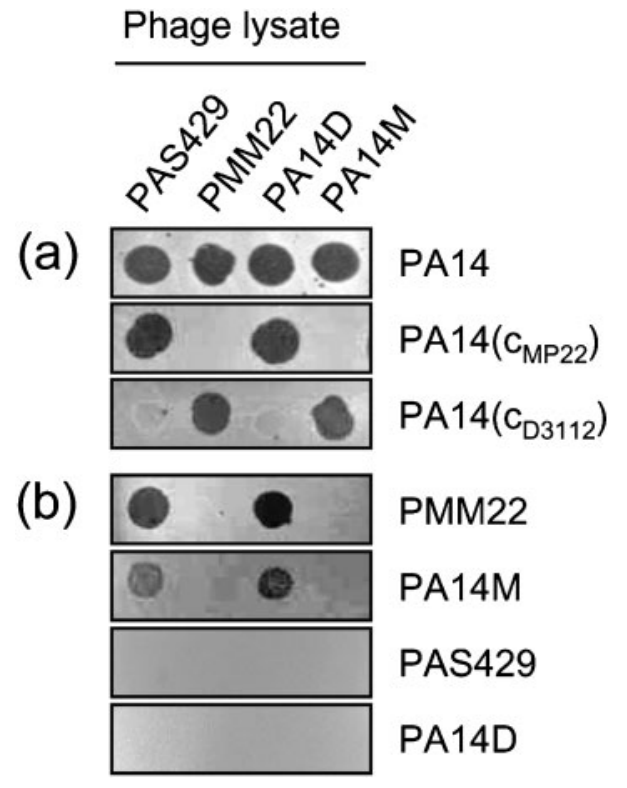

Fig. 7. Expression of $\mathrm{c}$ repressor and superinfection between MP22 and D3112. (a) Plaque formation on PA14 cells expressing c repressors. D3112 and MP22 phage lysates from $P$. aeruginosa lysogens (indicated at the top) were spotted onto lawns of PA14 cells (indicated on the right): PA14, PA14 cells containing pUCP18 vector only; PA14( $\left.\mathrm{C}_{\mathrm{MP} 22}\right)$ and PA14( $\left.\mathrm{c}_{\mathrm{D} 3112}\right), \mathrm{PA} 14$ cells expressing $\mathrm{c}$ repressor from MP22 and D3112, respectively on pUCP18. The zone of lysis on the plate indicates that the lysate was able to infect cells expressing the c repressor. (b) Plaque formation on $P$. aeruginosa lysogens. D3112 and MP22 phage lysates from $P$. aeruginosa lysogens (indicated at the top) were spotted onto lawns of $P$. aeruginosa lysogens (indicated on the right): MP22 lysogens (PMM22 and PA14M) and D3112 lysogens (PAS429 and PA14D). Plaque formation was measured by zone of clearing as in (a).

identity and biochemical properties of the gene(s) involved in the superinfectioin hierarchy warrant further investigation.

\section{Conclusions}

We have identified a temperate transposable phage, MP22, from a Korean clinical isolate (PMM22), which produces discernible plaques on the highly virulent $P$. aeruginosa strain PA14. MP22 has a $\lambda$-like morphology (Fig. 1) and a double-stranded DNA genome of $36 \mathrm{~kb}$ with variable host DNA fragments attached to the genome ends. Similar to several other phages, MP22 requires TFP for adsorption and subsequent infection of $P$. aeruginosa (Fig. 2). Complete genome sequencing of MP22 reveals that it is closely related to D3112, which belongs to the Siphoviridae, with $\mathrm{Mu}$-like characteristics. Comparison of the genomic organization and putative coding regions between MP22 and D3112 shows a remarkable overall similarity and synteny (Figs 3 and 4). Nevertheless, these two phages exhibit several significant differences, including ORFs with significantly lower protein sequence similarity (c repressor, Gam, capsid and tail proteins), and several mutually exclusive ORFs (Fig. 4). The D3112 genome has a few more genes, a slightly larger genome size and mosaicism (Fig. 4, Table 1). By careful comparison of the nucleotide sequences of MP22 and D3112 and a search for programmed frameshifts, we were able to identify and correct several annotation errors in the D3112 genome sequence (Fig. 5).

We have also found a marked difference in the second helix of the putative helix-turn-helix motif in the MP22 and D3112 c repressors (Fig. 6), which, we propose, prevents cross-immunity to superinfection by the non-cognate phage (Fig. 7). Interestingly, our observation of a superinfection hierarchy between lysogens suggests that one or more genes unique to D3112 can act either independently or in conjunction with $\mathrm{c}$ repressor to provide crossimmunity to superinfection by MP22. It will be interesting to uncover the factors involved in the superinfection hierarchy in terms of the molecular mechanisms governing phage repressor-operator interaction and phage evolution.

Further molecular biological studies based on the comparative genomic and proteomic analyses of phages MP22 and D3112 will be useful to elucidate the biogenesis, function and evolution of these related phages. They will also provide essential information for the development of new phage vectors and therapeutic agents to control infection by $P$. aeruginosa (Darzins \& Casadaban, 1989a; Groisman \& Casadaban, 1987; Hagens et al., 2004).

\section{ACKNOWLEDGEMENTS}

This work was supported by the 21C Frontier Microbial Genomics and Applications Center Program from the Korean Ministry of Science and Technology to Y.-H. C. (MG05-0104-05-0) and the Seoul R\&BD program. Y.-J.H., I.-Y.C. and K. B. C. were the recipients of BK21 Fellowships from the Korean Ministry of Education and Human Resources Development.

\section{REFERENCES}

Bibb, M. J., Findlay, P. R. \& Johnson, M. W. (1984). The relationship between base composition and codon usage in bacterial genes and its use for the simple and reliable identification of protein-coding sequences. Gene 30, 157-166.

Bodey, G. P., Bolivar, R., Fainstein, V. \& Jadeja, L. (1983). Infections caused by Pseudomonas aeruginosa. Rev Infect Dis 5, 279-313.

Boles, B. R., Thoendel, M. \& Singh, P. K. (2004). Self-generated diversity produces "insurance effects" in biofilm communities. Proc Natl Acad Sci U S A 101, 16630-16635.

Bradley, D. E. (1967). Ultrastructure of bacteriophage and bacteriocins. Bacteriol Rev 31, 230-314.

Bradley, D. E. (1973a). A pilus-dependent Pseudomonas aeruginosa bacteriophage with a long noncontractile tail. Virology 51, 489-492.

Bradley, D. E. (1973b). The length of the filamentous Pseudomonas aeruginosa bacteriophage Pf. J Gen Virol 20, 249-252. 
Braid, M. D., Silhavy, J. L., Kitts, C. L., Cano, R. J. \& Howe, M. M. (2004). Complete genomic sequence of bacteriophage B3, a Mu-like phage of Pseudomonas aeruginosa. J Bacteriol 186, 6560-6574.

Budzik, J. M., Rosche, W. A., Rietsch, A. \& O'Toole, G. A. (2004). Isolation and characterization of a generalized transducing phage for Pseudomonas aeruginosa strains PAO1 and PA14. J Bacteriol 186, 3270-3273.

Byrne, M. \& Kropinski, A. M. (2005). The genome of the Pseudomonas aeruginosa generalized transducing bacteriophage F116. Gene 346, 187-194.

Choi, K. H., Kumar, A. \& Schweizer, H. P. (2006). A 10-min method for preparation of highly electrocompetent Pseudomonas aeruginosa cells: application for DNA fragment transfer between chromosomes and plasmid transformation. J Microbiol Methods 64, 391-397.

Costerton, J. W., Stewart, P. S. \& Greenberg, E. P. (1999). Bacterial biofilms: a common cause of persistent infections. Science 284, 1318-1322.

Darling, A. C., Mau, B., Blattner, F. R. \& Perna, N. T. (2004). Mauve: multiple alignment of conserved genomic sequence with rearrangements. Genome Res 14, 1394-1403.

Darzins, A. \& Casadaban, M. J. (1989a). Mini-D3112 bacteriophage transposable elements for genetic analysis of Pseudomonas aeruginosa. J Bacteriol 171, 3909-3916.

Darzins, A. \& Casadaban, M. J. (1989b). In vivo cloning of Pseudomonas aeruginosa genes with mini-D3112 transposable bacteriophage. J Bacteriol 171, 3917-3925.

Daw, M. A. \& Falkiner, F. R. (1996). Bacteriocins: nature, function and structure. Micron 27, 467-479.

Drenkard, E. \& Ausubel, F. M. (2002). Pseudomonas biofilm formation and antibiotic resistance are linked to phenotypic variation. Nature 416, 740-743.

Govan, J. R. \& Deretic, V. (1996). Microbial pathogenesis in cystic fibrosis: mucoid Pseudomonas aeruginosa and Burkholderia cepacia. Microbiol Rev 60, 539-574.

Groisman, E. A. \& Casadaban, M. J. (1987). In vivo DNA cloning with a mini-Mu replicon cosmid and a helper lambda phage. Gene 51, 77-84.

Hagens, S., Habel, A., von Ahsen, U., von Gabain, A. \& Blasi, U. (2004). Therapy of experimental Pseudomonas infections with a nonreplicating genetically modified phage. Antimicrob Agents Chemother 48, 3817-3822.

Heo, Y.-J., Ko, K. S., Song, J.-H. \& Cho, Y.-H. (2005). Profiling pyocins and competitive growth advantages in various Pseudomonas aeruginosa strains. J Microbiol Biotechnol 15, 1368-1376.

Heo, Y.-J., Chung, I.-Y., Choi, K. B. \& Cho, Y.-H. (2007). R-type pyocin is required for competitive growth advantage between Pseudomonas aeruginosa strains. J Microbiol Biotechnol 17, 180-185.

Hoang, T. T., Karkhoff-Schweizer, R. R., Kutchma, A. J. \& Schweizer, H. P. (1998). A broad-host-range Flp-FRT recombination system for site-specific excision of chromosomally-located DNA sequences: application for isolation of unmarked Pseudomonas aeruginosa mutants. Gene 212, 77-86.

Hoang, T. T., Kutchma, A. J., Becher, A. \& Schweizer, H. P. (2000) Integration-proficient plasmids for Pseudomonas aeruginosa: sitespecific integration and use for engineering of reporter and expression strains. Plasmid 43, 59-72.

Isono, K., Mclninch, J. D. \& Borodovsky, M. (1994). Characteristic features of the nucleotide sequences of yeast mitochondrial ribosomal protein genes as analyzed by computer program GeneMark. DNA Res $1,263-269$.
Kielhofner, M., Atmar, R. L., Hamill, R. J. \& Musher, D. M. (1992). Lifethreatening Pseudomonas aeruginosa infections in patients with human immunodeficiency virus infection. Clin Infect Dis 14, 403-411.

Kropinski, A. M. (2000). Sequence of the genome of the temperate, serotype-converting, Pseudomonas aeruginosa bacteriophage D3. J Bacteriol 182, 6066-6074.

Krylov, V. N., Akhverdian, V. Z., Bogush, V. G., Khrenova, E. A. \& Reulets, M. A. (1985). Modular structure of the genes of phagestransposons of Pseudomonas aeruginosa. Genetika 21, 724-734.

Levin, M. E., Hendrix, R. W. \& Casjens, S. R. (1993). A programmed translational frameshift is required for the synthesis of a bacteriophage lambda tail assembly protein. J Mol Biol 234, 124-139.

Lowe, T. M. \& Eddy, S. R. (1997). tRNAscan-SE: a program for improved detection of transfer RNA genes in genomic sequence. Nucleic Acids Res 25, 955-964.

Ma, J., Campbell, A. \& Karlin, S. (2002). Correlations between ShineDalgarno sequences and gene features such as predicted expression levels and operon structures. J Bacteriol 184, 5733-5745.

Marrs, C. F. \& Howe, M. M. (1990). Kinetics and regulation of transcription of bacteriophage Mu. Virology 174, 192-203.

Pabo, C. O. \& Sauer, R. T. (1992). Transcription factors: structural families and principles of DNA recognition. Annu Rev Biochem 61, 1053-1095.

Rahme, L. G., Ausubel, F. M., Cao, H., Drenkard, E., Goumnerov, B. C., Lau, G. W., Mahajan-Miklos, S., Plotnikova, J., Tan, M. W. \& other authors (2000). Plants and animals share functionally common bacterial virulence factors. Proc Natl Acad Sci U S A 97, 8815-8821.

Rohwer, F. \& Edwards, R. (2002). The Phage Proteomic Tree: a genome-based taxonomy for phage. J Bacteriol 184, 4529-4535.

Roncero, C., Darzins, A. \& Casadaban, M. J. (1990). Pseudomonas aeruginosa transposable bacteriophages $\mathrm{D} 3112$ and $\mathrm{B} 3$ require pili and surface growth for adsorption. J Bacteriol 172, 1899-1904.

Salmon, K. A., Freedman, O., Ritchings, B. W. \& DuBow, M. S. (2000). Characterization of the lysogenic repressor (c) gene of the Pseudomonas aeruginosa transposable bacteriophage D3112. Virology 272, 85-97.

Sambrook, J., Fritsch, E. F. \& Maniatis, T. (1989). Molecular Cloning: a Laboratory Manual, 2nd edn. Cold Spring Harbor, NY: Cold Spring Harbor Laboratory.

Shine, J. \& Dalgarno, L. (1975). Determinant of cistron specificity in bacterial ribosomes. Nature 254, 34-38.

Sulakvelidze, A., Alavidze, Z. \& Morris, J. G., Jr (2001). Bacteriophage therapy. Antimicrob Agents Chemother 45, 649-659.

Summer, E. J., Gonzalez, C. F., Carlisle, T., Mebane, L. M., Cass, A. M., Savva, C. G., LiPuma, J. \& Young, R. (2004). Burkholderia cenocepacia phage $\mathrm{BcepMu}$ and a family of $\mathrm{Mu}$-like phages encoding potential pathogenesis factors. J Mol Biol 340, 49-65.

Thompson, J. D., Higgins, D. G. \& Gibson, T. J. (1994). CLUSTAL W: improving the sensitivity of progressive multiple sequence alignment through sequence weighting, position-specific gap penalties and weight matrix choice. Nucleic Acids Res 22, 4673-4680.

Wang, I. N., Smith, D. L. \& Young, R. (2000). Holins: the protein clocks of bacteriophage infections. Annu Rev Microbiol 54, 799-825.

Wang, P. W., Chu, L. \& Guttman, D. S. (2004). Complete sequence and evolutionary genomic analysis of the Pseudomonas aeruginosa transposable bacteriophage D3112. J Bacteriol 186, 400-410.

Xu, J., Hendrix, R. W. \& Duda, R. L. (2004). Conserved translational frameshift in dsDNA bacteriophage tail assembly genes. Mol Cell 16, $11-21$.

Edited by: M. A. Kertesz 